\title{
A Novel Method of DS Evidence Theory for Multi-Sensor Conflicting Information
}

\author{
Fang Liu' ${ }^{1}$ Yanxue Wang ${ }^{2}$ \\ 1 School of Mechanical and Electrical Engineering, Guilin University of Electronic Technology, \\ Guilin 541004, PR. China liudhuanglf@gmail.com \\ 2 School of Mechanical-Electronic and Vehicle Engineering, Beijing University of Civil Engineering \\ and Architecture, Beijing 100044, PR. China, yan.xue.wang@gmail.com
}

Keywords: multi-sensor fusion; evidential conflict; belief entropy; fuzzy preference relations; Dempster-Shafer evidence theory

\begin{abstract}
The multi-sensor data fusion technique plays a significant role in fault diagnosis and in a variety of applications. Conflict management is an open issue in Dempster-Shafer evidence theory. In this paper, a novel multi-sensor data fusion approach is proposed based on the spectral angle cosine function of evidence, belief entropy and group decision fuzzy preference relation analysis. The numerical simulation analyses demonstrate that the improved DS evidence theory available in this paper overcomes the limitations of conventional DS evidence theory, and can realizes more reliable fusion with multi-sensor conflicting information compared to the existing methods.
\end{abstract}

\section{Introduction}

With the development of expert systems, uncertain reasoning plays an increasingly important role in the intelligent systems. A number of uncertainty management approaches in uncertain reasoning systems have been developed. In these frameworks, Dempster-Shafer evidence theory (DST) is widely used for its flexibility and effectiveness in modelling both uncertainty and imprecision. In DST, the Dempster's rule of combination is used to implement the evidence combinations, which is located at the pivotal position. The necessary condition for using Dempster's rule is that all the combined bodies of evidence be distinct and reliable. However, in many real applications, the condition is not always fulfilled. Accordingly, counterintuitive results perhaps obtained by classical Dempster's rule of combination when dealing with unreliable sources of evidence, especially when there is high conflict among bodies of evidence.

To address this issue, some researchers have paid their attentions to Dempster's rule of combination and conflict management. They are divided into two types of methodologies. One is involves modifying Dempster's combination rule, while the second type involves pre-processing the bodies of evidence. The main research works for the first type include the combination rule presented by Yager [1]. But the modification of the combination rule often destructs the properties, like the commutativity and associativity. Therefore, many researchers study the ontologies of evidence to resolve the problem of highly conflict, which is the second type. The main research works for the second type include the simple average approach of the bodies of evidence proposed by Murphy [2], the weighted average of the masses based on the evidence distance presented by Deng et al. [3] and the cosine theorem-based method proposed by Zhang et al. [4]. However, the effect of evidence's uncertainty itself on the weight was overlooked.

In this paper, therefore, a novel multi-sensor data fusion method is proposed, which is a hybrid methodology in terms of the angle cosine, belief entropy and fuzzy preference relation analysis. The proposal considers the similarity among the pieces of evidence, the uncertainty measure of the evidence on the influence of the weight, so that it can obtain more appropriately weighted average evidence before using Dempster's combination rule. And which can solve the data conflict. 


\section{Basic Concept Reviewed}

\subsection{Dempster-Shafer Evidence Theory}

A frame of discernment (FOD) is a set of mutually exclusive and collectively exhaustive events, indicated by $\Theta=\left\{\mathrm{H}_{1}, \mathrm{H}_{2}, \ldots, \mathrm{H}_{n}\right\}$. The power set of $\Theta$ is denoted as $2^{\Theta}$. A mapping $m: 2^{\Theta} \rightarrow[0,1]$ is a mass function, also called basic probability assignment (BPA), defined on $\Theta$, if it satisfies

$$
m(\varnothing)=0, \quad \sum_{A \subseteq \Theta} m(A)=1
$$

Associated with each BPA, the belief function Bel and plausibility function $\mathrm{Pl}$ are defined as

$$
\begin{gathered}
\operatorname{Pl}(A)=1-\operatorname{Bel}(\bar{A})=\sum_{B \cap A \neq \varnothing} m(B) \\
\operatorname{Bel}(A)=\sum_{B \subseteq A} m(B)
\end{gathered}
$$

where $\bar{A}=\Omega-A$. Obviously, $\operatorname{Pl}(A) \geq \operatorname{Bel}(A)$, for each $A \subseteq \Theta$.

In belief function theory, two independent BPAs can be combined by Dempster's rule [5] of combination, denoted by $m=m_{1} \oplus m_{2}$ :

$$
m(A)= \begin{cases}\frac{1}{1-K} \sum_{B \cap C=A} m_{1}(B) m_{2}(C) & A \neq \varnothing \\ 0 & A=\varnothing\end{cases}
$$

with

$$
K=\sum_{B \cap C=\Theta} m_{1}(B) m_{2}(C)
$$

\subsection{Spectral Angle Cosine Function}

The spectral angle cosine function is calculate the generalized angle to determine the similarity between evidence. Employing the spectral angle cosine function, the similarity degree between evidence $m_{i}, m_{j}$ is

$$
S_{i j}=\operatorname{sim}\left(m_{i}, m_{j}\right)=\frac{\sum_{t=1}^{M} m_{i t} \cdot m_{j t}}{\sqrt{\sum_{t=1}^{M} m^{2}{ }_{i t}} \cdot \sqrt{\sum_{t=1}^{M} m^{2}{ }_{j t}}}
$$

Then, the support degree and credibility degree of evidence $m_{i}$ can be separately defined by $s_{i j}$

$$
\begin{gathered}
\sup \left(m_{i}\right)=\sum_{j=1,}^{N} s_{i j} \\
\operatorname{crd}(m)=\frac{\sup \left(m_{i}\right)}{\operatorname{Max}\left(\sup \left(m_{i}\right)\right)}
\end{gathered}
$$

It's important to note that the algorithm adds up all the elements in a row, including the elements on the diagonal [4]. This is different from Deng's method [3].

\subsection{Belief Entropy}

A belief entropy, called the Deng entropy, was first proposed by Deng [6], and has been applied in various fields. Deng entropy is proposed to measure the uncertainty of BPA. It is a generalized Shannon entropy. The basic concepts and definitions are introduced below.

Let A be a proposition of the basic probability assignment (BPA) on the frame of discernment $\Theta$; the Deng entropy $E_{d}(m)$ of the BPA is defined as follows:

$$
E_{d}(m)=-\sum_{A \subseteq \Theta} m(A) \log _{2} \frac{m(A)}{2^{|A|}-1}
$$

Set $A_{i}$ is a proposition of BPA, $\left|A_{i}\right|$ is the potential of $A_{i}$. If there is only one element in each proposition, it degenerates into Shannon entropy 


$$
E_{d}=-\sum_{A \subseteq \Theta} m(A) \log _{2} \frac{m(A)}{2^{|A|}-1}=-\sum_{A \subseteq \Theta} m(A) \log _{2} m(A)
$$

\subsection{Fuzzy Preference Relations}

Fuzzy preference relations play a fundamental part in many decision-making processes, and they were first presented by Tanino [7]. The concepts of fuzzy preference relations as below.

Let $P$ be a fuzzy preference relation for the set $A$ of alternatives, $A=\left\{A_{1}, A_{2}, \ldots, A_{n}\right\}$, shown as follows:

$$
P=\left(p_{i j}\right)_{n \times n}=\left[\begin{array}{cccc}
0.5 & p_{12} & \cdots & p_{1 n} \\
p_{21} & 0.5 & \cdots & p_{2 n} \\
\vdots & \vdots & \ddots & \vdots \\
p_{n 1} & p_{n 2} & \cdots & 0.5
\end{array}\right]
$$

where $p_{i j}$ denotes the degree of preference of alternative $A_{i}$ over alternative $A_{j}, p_{i j} \in[0,1]$. If $P$ is a complete fuzzy preference relation which satisfies the following additive consistency properties for all $i, j$ and $k$ :

$$
\begin{aligned}
& p_{i j}+p_{j i}=1 \\
& p_{i i}=0.5 \\
& p_{i k}=p_{i j}+p_{j k}-0.5
\end{aligned}
$$

where $1 \leq i \leq n, 1 \leq j \leq n$ and $1 \leq k \leq n$, then $P$ is called an additive consistent fuzzy preference relation.

Subsequently, Chen [8] proposed a new method to solve the problem about the consistency of the order in fuzzy preference relations.

Step 1: Construct the consistency matrix $\bar{P}^{q}$ for expert $E_{q}$, and show as follows:

$$
\bar{P}^{q}=\left(\bar{p}_{i k}\right)_{n \times n}=\left(\frac{1}{2 n} \sum_{j=1}^{n}\left(P_{i j}^{q}-P_{j i}^{q}+P_{j k}^{q}-P_{i j}^{q}\right)+0.5\right)_{n \times n}
$$

Step 2: Calculate the boundary constant $b_{q}$ and the consistency degree $c_{q}$ for expert $E_{q}$, shown as below:

$$
\begin{aligned}
& s_{i}^{q}=\frac{1}{n} \sum_{j=1}^{n} \bar{P}_{i j}^{q} \\
& m a_{q}=\operatorname{Max}\left(s_{i}^{q} \mid 1 \leq i \leq n\right) \\
& m i_{q}=\operatorname{Min}\left(s_{i}^{q} \mid 1 \leq i \leq n\right) \\
& b_{q}=\frac{1}{2 \times \operatorname{Max}\left(0.5,\left(m a_{q}-m i_{q}\right)\right)} \\
& c_{q}=1-\frac{2}{n(n-2)} \sum_{i=1}^{n} \sum_{k=1, k \neq i}^{n}\left|P_{i k}^{q}-\bar{P}_{i k}^{q}\right|
\end{aligned}
$$

where $s_{i}^{q}$ is the average value of alternative $A_{i}, m a_{q}$ is the maximum value of all $s_{i}^{q}, m i_{q}$ is the minimum value of all $s_{i}^{q}, b_{q} \in[0,1], 1 \leq i \leq n, 1 \leq k \leq n$ and $1 \leq q \leq m$.

Step 3: Construct the modified consistency matrix $\bar{P}^{q}$ for expert $E_{q}$, shown as follows:

$$
\tilde{P}^{q}=\left(\tilde{P}_{i k}^{q}\right)_{n \times n}=\left(\bar{P}_{i k}^{q} \times d_{q}+\frac{1}{2}\left(1-d_{q}\right)\right)_{n \times n}
$$

where $d_{q}$ denotes the modified constant for expert $E_{q}$, and $d_{q}=b_{q} \times c_{q}, d_{q} \in[0,1], \bar{P}_{i i}^{q}=0.5$.

Step 4: Calculate the ranking value $R\left(A_{i}\right)$ of alternative $A_{i}$, shown as below:

$$
R\left(A_{i}\right)=\frac{2}{n^{2}-n} \sum_{j=1, j \neq i}^{n} \tilde{P}_{i j}^{q}
$$

where $\sum_{i=1}^{n} R\left(A_{i}\right)=1$. The larger the value of $R\left(A_{i}\right)$, the better the ranking order of alternative $A_{i}$. 


\section{The Proposed Method}

Combined with the above instructions, the detailed process of the proposed method is as follows:

\subsection{Calculate the Support Degree of the Evidence}

Step 1: The angle cosine measure $s_{i j}$ between the BPAs $m_{i}$ and $m_{j}$ can be obtained by (6);

Step 2: The support degree of BPA $m_{i}$ is defined as (7):

Step 3: The support degree of the BPA $m_{i}$ is normalized as below, which is denoted as (8).

\subsection{The Credibility Value of the Evidence}

We take advantage of the fuzzy preference relations analysis based on the belief entropy to indicate the relative credibility preference among the pieces of evidence.

Step 1: The belief entropy of the BPA $m_{i}$ is calculated by (9), in order to avoid allocating zero weight to evidence, we use $D_{i}$ for measuring the uncertainty of the BPA $m_{i}$ as below:

$$
D_{i}=e^{E_{d}\left(m_{i}\right)}, 1 \leq i \leq k
$$

Step 2: The normalized volume of the BPA $m_{i}$ is as follow, which is $\bar{V}_{i}$ :

$$
\bar{V}_{i}=\frac{V_{i}}{\sum_{i=1}^{k} V_{i}}, 1 \leq i \leq k
$$

Step 3: The fuzzy preference relation matrix $P=\left(P_{i j}\right)_{k \times k}$, which can be constructed as below:

$$
P_{i j}=\frac{\operatorname{Var}_{i}}{\operatorname{Var}_{i}+\operatorname{Var}_{j}}, \quad P_{j i}=\frac{\operatorname{Var}_{j}}{\operatorname{Var}_{i}+\operatorname{Var}_{j}}
$$

where the variance of entropy for the BPA $m_{i}$ will be calculated as follows:

$$
\operatorname{Var}_{i}=\operatorname{Var}\left(\left\{\bar{V}_{1}, \bar{V}_{1}, \ldots, \bar{V}_{i-1}, \bar{V}_{i+1}, \ldots, \bar{V}_{k},\right\}\right)
$$

Step 4: Based on the obtained fuzzy preference relation matrix, the consistency matrix $\bar{P}^{q}$ and the modified consistency matrix $\tilde{P}^{q}$ can be constructed by (15)-(21).

Step 5: With the modified consistency matrix $\tilde{P}^{q}$, the credibility value of the BPA $m_{i}$ is defined based on (23).

\subsection{Fusion with the Weighted Average Evidence}

Step 1: Based on the credibility degree $c r d_{i}$, the normalized support degree of the BPA $m_{i}$ will be adjusted, denoted as $P S p_{i}$ :

$$
\operatorname{PSup}_{i}=\operatorname{crd}_{i} \times R_{i}
$$

Step 2: The final weight of the BPA $m_{i}$ is normalized as below:

$$
\bar{P} \operatorname{Sup}_{i}=\frac{\operatorname{PSup}_{i}}{\sum_{i=1}^{k} \operatorname{PSup}}
$$

Step 3: the weighted average evidence $W(m)$ can be obtained as follows:

$$
W(m)=\sum_{i=1}^{k} \bar{P} \operatorname{Sup}_{i} \times m_{i}
$$

Step 4: Using the weighted average evidence $W(m)$ and combined through Dempster's combination rule by (4) with $k-1$ times.

\section{EXPERIMENTAL VERIFICATION}

In order to demonstrate the effectiveness of the proposal, a numerical example is illustrated. And the proposal is applied to the fault diagnosis of a motor rotor, where the practical data in [3] are used for the comparison with the related method.

These sensor reports that are modeled as the BPAs are given in Table I, where the FOD is given by $\Theta=\{A, B, C\}$. 
table i. The Basic Probability Assignments (BPAs)

\begin{tabular}{|c|c|c|c|c|}
\hline $\mathbf{B P A}$ & $\{\mathbf{A}\}$ & $\{\mathbf{B}\}$ & $\{\mathbf{C}\}$ & $\{\mathbf{A}, \mathbf{C}\}$ \\
\hline$m_{1}$ & 0.41 & 0.29 & 0.30 & 0.00 \\
\hline$m_{2}$ & 0.00 & 0.90 & 0.10 & 0.00 \\
\hline$m_{3}$ & 0.58 & 0.07 & 0.00 & 0.35 \\
\hline$m_{4}$ & 0.55 & 0.10 & 0.00 & 0.35 \\
\hline$m_{5}$ & 0.60 & 0.10 & 0.00 & 0.30 \\
\hline
\end{tabular}

Step 1: Construct the similarity matrix $\operatorname{SIM}=\left(s_{i j}\right)_{k \times k}$ :

$$
\operatorname{SIM}=\left(\begin{array}{ccccc}
1 & 0.5493 & 0.6479 & 0.6596 & 0.6931 \\
0.5493 & 1 & 0.1022 & 0.1507 & 0.1465 \\
0.6479 & 0.1022 & 1 & 0.9985 & 0.9959 \\
0.6596 & 0.1507 & 0.9985 & 1 & 0.9948 \\
0.6931 & 0.1465 & 0.9959 & 0.9948 & 1
\end{array}\right)
$$

Step 2: Normalize the support degree of the BPA $m_{i}$ as follows:

$$
\operatorname{crd}_{i}=[0.9268,0.5088,0.9776,0.9930,1] '
$$

Step 3: Measure the information volume of the BPA $m_{i}$ and normalize as follow:

$$
\bar{V}_{i}=[0.1916,0.0639,0.2442,0.2652,0.2351]
$$

Step 4: Construct the modified consistency matrix $\tilde{P}^{q}$ as follows:

$$
\tilde{P}=\left(\begin{array}{ccccc}
0.5 & 0.9073 & 0.5205 & 0.5487 & 0.5124 \\
0.0927 & 0.5 & 0.1132 & 0.1414 & 0.1051 \\
0.4795 & 0.8868 & 0.5 & 0.5282 & 0.4919 \\
0.4513 & 0.8586 & 0.4718 & 0.5 & 0.4637 \\
0.4876 & 0.8949 & 0.5081 & 0.5363 & 0.5
\end{array}\right)
$$

Step 5: Calculate the credibility value of $m_{i}$ as below:

$$
R_{i}=[0.2489,0.0452,0.2386,0.2245,0.2427]^{\prime}
$$

Step 6: The final weight of $m_{i}$ is normalized as below:

$$
\overline{\mathrm{P} S u p}{ }_{i}=[0.2421,0.0242,0.2449,0.2341,0.2548]^{\prime}
$$

\begin{tabular}{|c|c|c|c|c|c|c|}
\hline Evidence & Method & $\{A\}$ & $\{\mathbf{B}\}$ & $\{c\}$ & $\{\mathrm{AC}\}$ & Target \\
\hline \multirow{7}{*}{$m_{1}, m_{2}, m_{3}$} & $\begin{array}{c}\text { Dempster } \\
\text { [5] }\end{array}$ & 0 & 0.6350 & 0.3650 & 0 & B \\
\hline & Murphy [2] & 0.4939 & 0.4180 & 0.0792 & 0.0090 & A \\
\hline & $\begin{array}{l}\text { Deng et al. } \\
\text { [3] }\end{array}$ & 0.4974 & 0.4054 & 0.0888 & 0.0084 & A \\
\hline & $\begin{array}{l}\text { Zhang et al. } \\
\text { [4] }\end{array}$ & 0.5681 & 0.3319 & 0.0929 & 0.0084 & A \\
\hline & \multicolumn{2}{|c|}{$\begin{array}{l}\text { Proposed method } \\
\mathbf{0 . 8 3 0 8}\end{array}$} & 0.0532 & 0.1046 & 0.0115 & A \\
\hline & $\begin{array}{c}\text { Dempster } \\
\text { [5] }\end{array}$ & 0 & 0.3321 & 0.6679 & 0 & $\mathrm{C}$ \\
\hline & Murphy [2] & 0.8362 & 0.1147 & 0.0410 & 0.0081 & A \\
\hline$m_{1}, m_{2}, m_{3}, m_{4}$ & Deng et al. & 0.9089 & 0.0444 & 0.0379 & 0.0089 & A \\
\hline
\end{tabular}

Step 7: Compute weighted average evidence as below:

$$
\begin{gathered}
m(\{A\})=0.5229, m(\{B\})=0.1580, m(\{C\})=0.0751, \\
m(\{A, C\})=0.2441
\end{gathered}
$$
times.

Step 8: Combine the weighted average evidence by utilizing Dempster's rule of combination four

TABLE II. Combination results of the evidence in terms of different combination rules. 


\begin{tabular}{|c|c|c|c|c|c|c|}
\hline \multicolumn{7}{|c|}{$[3]$} \\
\hline & $\begin{array}{c}\text { Zhang et al. } \\
\text { [4] }\end{array}$ & 0.9142 & 0.0395 & 0.0399 & 0.0083 & A \\
\hline & \multicolumn{2}{|c|}{$\begin{array}{l}\text { Proposed method } \\
\mathbf{0 . 9 5 3 5}\end{array}$} & 0.0046 & 0.0334 & 0.0085 & A \\
\hline \multirow{5}{*}{$m_{1}, m_{2}, m_{3}, m_{4}, m_{5}$} & $\begin{array}{c}\text { Dempster } \\
{[5]}\end{array}$ & 0 & 0.1422 & 0.8578 & 0 & $\mathrm{C}$ \\
\hline & Murphy [2] & 0.9620 & 0.0210 & 0.0138 & 0.0032 & A \\
\hline & $\begin{array}{l}\text { Deng et al. } \\
\text { [3] }\end{array}$ & 0.9820 & 0.0039 & 0.0107 & 0.0034 & A \\
\hline & $\begin{array}{l}\text { Zhang et al. } \\
\text { [4] }\end{array}$ & 0.9820 & 0.0034 & 0.0115 & 0.0032 & A \\
\hline & \multicolumn{2}{|c|}{$\begin{array}{l}\text { Proposed method } \\
\mathbf{0 . 9 8 8 6}\end{array}$} & 0.0004 & 0.0091 & 0.0032 & A \\
\hline
\end{tabular}

As shown in Table II, the proposed method is efficient in dealing with the conflicting pieces of evidence with better convergence. The reason is that the proposal not only makes use of the function of evidence similarity to obtain the evidence's support degree, but also adopts the fuzzy preference relations analysis based on the belief entropy to measure the relative credibility preference among the pieces of evidence. Therefore, the unreliable evidence's weight is decreased, so that its negative effect can be relieved on the final fusing results compared to other methods.

\section{Conclusions}

In this paper, on account of the support degree among the pieces of evidence, the uncertainty measure of the evidence and the effect of the relative credibility of evidence on the weight, a novel method for multi-sensor data fusion was proposed. The proposed method was a hybrid methodology by integrating the similarity of evidence, belief entropy and fuzzy preference relation analysis. Through a numerical example, it was illustrated that the proposal was more effective and feasible than other related methods to handle the conflicting evidence combination problem under a multisensor environment with better convergence.

\section{Acknowledgment}

The financial sponsorship from the project of National Natural Science Foundation of China (61463010, 51475098 and 51405093) and Guangxi Natural Science Foundation (2016GXNSFFA380008) are gratefully acknowledged. It is also sponsored by Guangxi key laboratory of manufacturing system \& advanced manufacturing technology (15-140-30-001Z, 16380-12-004Z, 16-380-12-011K, and 14-045-15-006Z), Innovation Project of Guangxi Graduate Education (YCSW2017136) and basic ability promotion project for young and middle-aged teachers in Guangxi Province (Grant No. 2017KY0207).

\section{References}

[1] Yager, R.R. Entropy and specificity in a mathematical theory of evidence. Int. J. Gen. Syst. 1983, 9, 249-260.J. Clerk Maxwell, A Treatise on Electricity and Magnetism, 3rd ed., vol. 2. Oxford: Clarendon, 1892, pp.68-73.

[2] Murphy, C.K. Combining belief functions when evidence conflicts. Decis. Support Syst. 2000, 29, 1-9.K. Elissa, "Title of paper if known," unpublished.

[3] Deng,Y; Shi,W.;Zhu,Z.;Liu,Q. Combining belief function based on distance of evidence. Decis.Support Syst.2004,38,489-493

[4] Zhang,Z.;Liu,T;Chen,D.;Zhang,W. Novel algorithm for identifying and fusing conflicting data in wireless sensor networks. Sensors 2014,14,9562-9581. 
[5] Dempster, A.P. Upper and lower probabilities induced by a multivalued mapping. Ann. Math. Stat. 1967, 38,325-339.

[6] Deng, Y. Deng entropy. Chaos Solitons Fractals 2016, 91, 549-553.

[7] Tanino, T. Fuzzy preference orderings in group decision making. Fuzzy Sets Syst. 1984, 12, 117-131.

[8] Chen, S. M., Lin, T. E., \& Lee, L. W. Group decision making using incomplete fuzzy preference relations based on the additive consistency and the order consistency. Information Sciences, 2014, 259, 1-15 